\title{
Hydroxytamoxifen interaction with human erythrocyte membrane and induction of permeabilization and subsequent hemolysis
}

\author{
M.M. Cruz Silva ${ }^{\mathrm{a}}$, V.M.C. Madeira ${ }^{\mathrm{b}}$, L.M. Almeida ${ }^{\mathrm{a}, \mathrm{b}}$, J.B.A. Custódio ${ }^{\mathrm{a}, \mathrm{b}, *}$ \\ ${ }^{a}$ Laboratório de Bioquímica, Faculdade de Farmácia, 3000 Coiombra, Portugal \\ ${ }^{\mathrm{b}}$ Centro de Neurociencias de Coimbra, Universidade de Coimbra, 3000 Coiombra, Portugal
}

Accepted 4 March 2001

\begin{abstract}
4-Hydroxytamoxifen (OHTAM) is the most active metabolite of the widely prescribed anticancer drug tamoxifen (TAM) used in breast cancer therapy. This work describes the effects of OHTAM on isolated human erythrocytes, using standardized test conditions, to check for a putative contribution to the TAM-induced hemolysis and to study basic mechanisms involved in the interaction of OHTAM with cell membranes. Incubation of isolated human erythrocytes with relatively high concentrations of OHTAM results in a concentration-dependent hemolysis, its hemolytic effect being about one-third of that induced by TAM. OHTAMinduced hemolysis is prevented by either $\alpha$-tocopherol $(\alpha-T)$ or $\alpha$-tocopherol acetate $(\alpha-T A c)$ and it occurs in the absence of oxygen consumption and hemoglobin oxidation, ruling out the oxidative damage of erythrocytes. However, OHTAM remarkably increases the osmotic fragility of erythrocytes, increasing the susceptibility of erythrocytes to hypotonic lysis. Additionally, the hemoglobin release induced by OHTAM is preceded by a rapid efflux of intracellular $\mathrm{K}^{+}$. Therefore, our data suggest that OHTAM-induced hemolysis does not contribute to TAM-induced hemolytic anemia and it is a much weaker toxic drug as compared with TAM. Moreover, at variance with the membrane disrupting effects of TAM, OHTAM promotes perturbation of the membrane's backbone region due to its strong binding to proteins with consequent formation of membrane paths of permeability to small solutes and retention of large solutes like hemoglobin, followed by osmotic swelling and cell lysis. The prevention of OHTAM-induced hemolysis by $\alpha$-T and $\alpha$-TAc is probably committed to the permeability sealing resulting from structural stabilization of membrane. (C) 2001 Elsevier Science Ltd. All rights reserved.
\end{abstract}

Keywords: 4-Hydroxytamoxifen; Tamoxifen; Human erythrocytes; Hemolysis; Oxidative stress; Osmotic swelling

\section{Introduction}

Tamoxifen (TAM) is a triphenylethylenic anti-estrogen commonly prescribed in the breast cancer therapy (Jordan, 1990) and potentially useful in the prevention of this malignant process (Nayfield et al., 1991).

Most studies indicated that the anticancer action of TAM is not solely mediated by the antagonism at the estrogen receptors (ER). Among the several mechanisms proposed, the interaction with biomembranes has been favoured (Koedijk et al., 1994). In fact, TAM that binds to proteins in a unspecific way (Oosbree et al.,

Abbreviations: AAPH, 2,2'-azobis(2-amidinopropane)dihydrochloride; $\alpha$-T, $\alpha$-tocopherol; $\alpha$-TAc, $\alpha$-tocopherol acetate; OHTAM, 4-hydroxytamoxifen; PBS, phosphate buffered saline; TAM, tamoxifen.

* Corresponding author. Tel.: + 351-239-852564; fax: + 351-239852569.

E-mail address: custodio@ci.uc.pt (J.B.A. Custódio).
1984) partitions in a greater extent in native membranes relatively to the respective lipid dispersions (Custódio et al., 1991), where it interacts with lipids (Custódio et al., 1993a) and proteins (Lopes et al., 1990; Custódio et al., 1996), explaining the modifications in the morphology and structure of breast tumor cell membranes (Sica et al., 1984). Moreover, TAM disrupts model (Vogel et al., 1995; Custódio et al., 1996) and mitochondrial membranes (Custódio et al., 1998) and its hemolytic effect in isolated human erythrocytes has been assigned to a structural perturbation of the membrane integrity caused by its strong accumulation within membranes (Cruz-Silva et al., 2000). Therefore, the TAM-induced structural disruption of biomembranes, as observed in different membrane systems, may contribute for its ERindependent anticancer activity and adverse effects, including hemolytic anemia (Ching et al., 1992; Thangaraju et al., 1995).

The metabolites of TAM transformation, mostly 4hydroxytamoxifen (OHTAM), have been reported to be 
pharmacologically active (Kemp et al., 1983), playing an important role in the therapeutic activity and sideeffects of TAM (Jordan, 1990). Actually, the anti-estrogenic and antiproliferative activities of OHTAM in vitro are much stronger as compared with TAM (Borgna and Rochefort, 1981; Etienne et al., 1989), but the pharmacological relevance of OHTAM has been questioned (McCague et al., 1990) due to its low serum concentrations (Lien et al., 1987; MacCallum et al., 1996). However, the strong affinity of OHTAM for biomembrane proteins (Custódio et al., 1991) and its higher partitioning in the lipid bilayer as compared with TAM (Custódio et al., 1991), perturbing the membrane lipid-protein interface (Custódio et al., 1993b) suggest that OHTAM may accumulate in tissues, reaching there the required effective concentrations for its cytotoxic effects, as described by Lien et al. (1991).

Therefore, the reports of hemolytic anemia caused by TAM (Ching et al., 1992; Thangaraju et al., 1995), the strong incorporation of OHTAM into biomembranes (Custódio et al., 1991) and the putative role of OHTAM in the multiple cellular effects of TAM (Jordan, 1990) prompted us to search for the effects of OHTAM on isolated erythrocytes, attempting to clarify the contribution of OHTAM to the hemolytic anemia caused by TAM and the basic mechanisms underlying the interaction of this metabolite with biomembranes. The use of standardized methods is helpful to compare the effects of OHTAM with data previously published (Cruz-Silva et al., 2000).

\section{Materials and methods}

\subsection{Chemicals}

4-Hydroxytamoxifen, tamoxifen and $\alpha$-tocopherol acetate $(\alpha-$ TAc) were purchased from Sigma Chemical Co (St Louis, MO, USA). 2,2'-Azobis(2-amidinopropane)dihydrochloride (AAPH) was obtained from Polysciences, Inc. (Warrington, PA, USA) and $\alpha$-tocopherol $(\alpha-T)$ from Fluka BioChemika (Switzerland). All the other chemicals were of research grade. Solutions were prepared in deionized ultra-pure water.

\subsection{Red blood cells preparation}

Heparinised human blood samples were centrifuged at $3000 \mathrm{rpm}$ for $10 \mathrm{~min}$. The sediment of erythrocytes was washed three times with 6 volumes of isotonic phosphate buffered saline (PBS) (150 mm NaCl, $10 \mathrm{~mm}$ sodium phosphate, $\mathrm{pH}$ 7.4) and the buffy coat was removed with each wash, according to previous reports (Urano et al., 1992; Dwight and Hendry, 1996; Koga et al., 1998). The retrieved cells were resuspended in PBS and the hematocrit determined. All the erythrocyte sus- pensions used were prepared daily from freshly collected blood obtained from 12 healthy donors (25-50 years old and not submitted to any drug treatment) who are working in our laboratory, as described elsewhere (Cruz-Silva et al., 2000).

\subsection{Hemolysis measurements}

The hemolysis studies were performed in erythrocyte suspensions $(4 \mathrm{ml})$ in PBS with the hematocrit at $0.33 \%$. OHTAM or TAM were added from stock ethanolic solutions $(2-4 \mu \mathrm{l})$ to the erythrocyte suspensions and incubated at $37^{\circ} \mathrm{C}$ in a shaking water-bath with magnetic stirring. The effects of $\alpha$-T and $\alpha$-TAc were assessed by pre-incubating the erythrocyte suspensions with these compounds at $37{ }^{\circ} \mathrm{C}$ for $2 \mathrm{~h}$, before the drug addition. A set of samples, after the pre-incubation period with the tocopherols, were washed three times with $10 \mathrm{ml}$ of PBS before the addition of OHTAM.

After the incubation periods, the erythrocyte suspensions were centrifuged at $3000 \mathrm{rpm}$ for $10 \mathrm{~min}$ and the hemolysis degree was estimated by visible spectroscopy at $540 \mathrm{~nm}$ from the hemoglobin released into the supernatant, as described elsewhere (Koga et al., 1998). The results were expressed as percent hemolysis. The absorbance of the supernatant in the absence of OHTAM was taken as zero hemolysis and the total hemolysis $(100 \%)$ was assigned when PBS was replaced by water containing identical volume of ethanol as used in the assays with OHTAM.

The osmotic fragility experiments were carried out according to standardized procedures (Takeuchi et al., 1989; Jackson et al., 1996) in phosphate buffered solutions (10 mm sodium phosphate, $\mathrm{pH}$ 7.4) with increasing concentrations of $\mathrm{NaCl}$ and a hematocrit of $0.33 \%$. After centrifugation at $3000 \mathrm{rpm}$ for $10 \mathrm{~min}$, the absorbance of supernatants was read at $540 \mathrm{~nm}$.

The $\mathrm{K}^{+}$leakage from erythrocytes was monitored with a $\mathrm{K}^{+}$ion-selective electrode. The total amount of $\mathrm{K}^{+}$was measured after the addition of $0.5 \%$ Triton $\mathrm{X}$ 100 to the erythrocyte suspensions.

\subsection{Erythrocytes oxidation measurements}

The erythrocyte hemolysis induced by OHTAM were followed by oxygen consumption and changes in hemoglobin absorption spectrum, as described elsewhere (Cruz-Silva et al., 2000).

The rate of oxygen consumption was measured with a Clark-type oxygen electrode (YSI model 5331, Yellow Spring Inst.), as previously described (Custódio et al., 1994). The reactions were carried out at $37{ }^{\circ} \mathrm{C}$ in a closed glass vessel with magnetic stirring and the hemolysis of erythrocyte suspension (hematocrit of $0.33 \%$ ) was induced by addition of $35 \mu \mathrm{M}$ OHTAM. After 90 min of OHTAM incubation, the free radicals generator 
AAPH (20 mM) was added and oxygen consumption was followed for another period of $60 \mathrm{~min}$. The oxygen consumption was calculated assuming that the $\mathrm{O}_{2}$ concentration in water was $177 \mathrm{nmol} / \mathrm{ml}$ at $37^{\circ} \mathrm{C}$.

In the course of incubation of erythrocyte suspensions with OHTAM and after addition of $20 \mathrm{~mm} \mathrm{AAPH}$, aliquots of the suspension were withdrawn, centrifuged at $3000 \mathrm{rpm}$ for $5 \mathrm{~min}$ and the spectrum of the released hemoglobin was recorded. The withdrawn aliquots were chilled prior to centrifugation to stop the thermal decomposition reaction of the azoinitiator. Hemoglobin oxidation was followed by the decay of the absorption spectrum in the range $450-650 \mathrm{~nm}$, using a Perkin-Elmer Lambda 6 UV/VIS spectrophotometer (Norwalk, USA).

The results are typical assays or represent the mean \pm S.D. of three independent experiments.

\section{Results}

\subsection{OHTAM-induced hemolysis}

The effects of OHTAM in isolated human erythrocytes were assayed as a function of incubation time and drug concentration in erythrocyte suspensions from different human donors (Fig. 1, lines 1-4). This figure shows the time-dependent hemolysis induced by $30 \mu \mathrm{M}$ OHTAM. Variations are observed in the susceptibility of samples to hemolysis depending on the blood donor, but an almost complete hemolysis is observed for all samples with $30 \mu \mathrm{M}$ OHTAM after 90 min of incubation, whereas $35 \mu \mathrm{M}$ OHTAM induces full hemolysis after this incubation time. As previously reported for TAM (Cruz-Silva et al., 2000), the sensitivity of erythrocytes to hemolysis induced by OHTAM is also lower for older subjects (Fig. 1, lines 3-4), according to earlier reports pointing out that the hemolysis extension decreases as a function of age as a consequence of increased membrane cholesterol (Araki and Rifkind, 1980). As OHTAM partitioning in biomembranes decreases as a function of cholesterol (Custodio et al., 1991), the different effects induced by OHTAM in diverse erythrocyte suspensions could be explained by the putative protection ascribed to membrane cholesterol. The hemolytic effect of OHTAM is also concentration dependent and total hemolysis is detected in all the erythrocyte samples when incubated with $35 \mu \mathrm{M}$ OHTAM, at $37^{\circ} \mathrm{C}$, for $90 \mathrm{~min}$ (Fig. 2).

The hemolytic action of OHTAM was also compared with the effects of TAM in the same erythrocyte samples, as shown in Fig. 2. TAM $(12.5 \mu \mathrm{M})$ induces complete hemolysis after $1 \mathrm{~h}$ of incubation, whereas the same concentration of OHTAM does not promote any significant effect and a total hemolysis is only achieved at $35 \mu \mathrm{M}$ OHTAM after $1.5 \mathrm{~h}$ of incubation. Identical experiments carried out with both drugs at $37^{\circ} \mathrm{C}$ for $1 \mathrm{~h}$ demonstrated that $12.5 \mu \mathrm{M}$ TAM induces total hemolysis whereas $35 \mu \mathrm{M}$ OHTAM promotes only about $70 \%$ hemolysis (data not shown). The combination of low concentrations of TAM $(<7.5 \mu \mathrm{M})$ with OHTAM $(<10$ $\mu \mathrm{M})$ does not change the effects observed with TAM alone, meaning that in vivo the interaction between these drugs will not cause increased hemolysis, in addition to the effect of TAM. Moreover, as described to TAM (Cruz-Silva et al., 2000), OHTAM induces the release of hemoglobin bound to the membrane, as evaluated by SDS-polyacrylamide gel electrophoresis, explaining the extra absorbance as compared to the total hemolysis induced by water (Fig. 2), also meaning that OHTAM changes the binding of cytosol and cytoskeleton proteins to band 3, the most abundant intrinsic protein of erythrocytes (Schuck and Schubert, 1991).

To clarify the potential mechanisms involved in the hemolytic effect of high concentrations of OHTAM, namely cellular oxidative stress or membrane structural perturbations, the protective effects of antioxidants and membrane stabilizers, for example $\alpha$-T and $\alpha$-TAc, were studied.

OHTAM-induced hemolysis is partially prevented by $\alpha-$ T (Fig. 3A) or $\alpha$-TAc (Fig. 3B) previously incubated with erythrocyte suspensions. When erythrocytes are washed after the pre-incubation period to avoid the interference of non-incorporated tocopherol compounds,

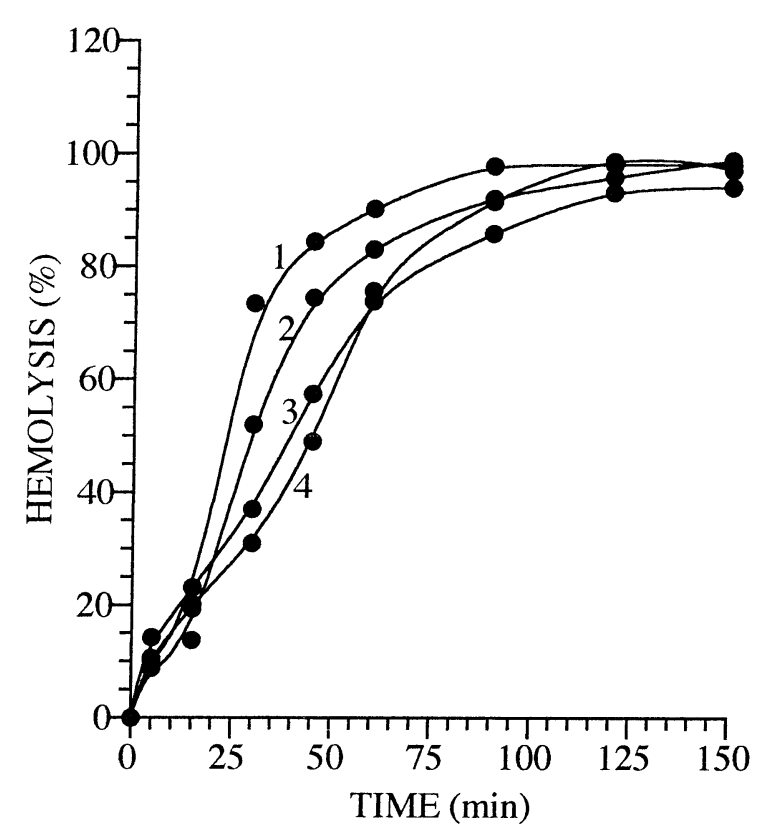

Fig. 1. OHTAM-induced hemolysis in human erythrocytes obtained from different donors (lines 1-4). Erythrocyte suspensions in phosphate buffer saline (PBS) $(0.33 \%$ hematocrit) were incubated with 30 $\mu \mathrm{M}$ OHTAM at $37^{\circ} \mathrm{C}$, as a function of time. After incubation with the drug, erythrocyte suspensions were centrifuged at $3000 \mathrm{rpm}$ for $10 \mathrm{~min}$ and hemolysis was estimated from the $540 \mathrm{~nm}$ absorbance of hemoglobin released into the supernatant. Data are expressed as percentage of total hemolysis induced by replacing the PBS by identical volume of water. 
the protection against OHTAM-induced hemolysis is nearly maintained as in unwashed erythrocytes (Fig. 3A,B). Therefore, these protective effects putatively result from the interaction of tocopherols with the erythrocyte membrane core (Shinitzky and Barenholz,

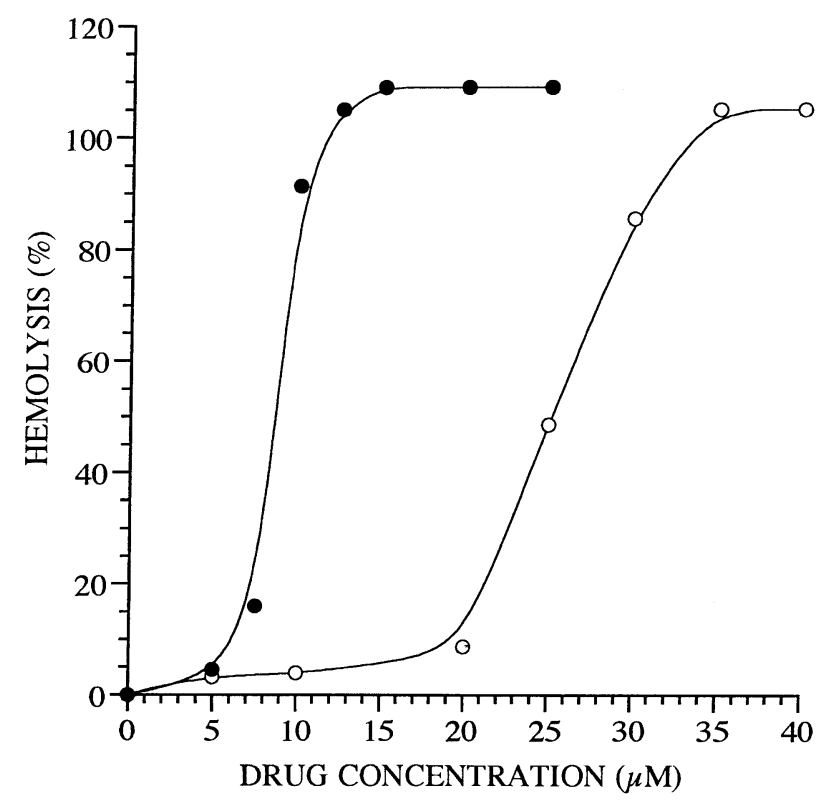

Fig. 2. Comparison of the hemolytic effects induced by TAM (O) and OHTAM $(\bigcirc)$ in the same erythrocyte suspension, as a function of drug concentration. Erythrocyte suspension in PBS ( $0.33 \%$ hematocrit) were incubated with different concentrations of either TAM at $37{ }^{\circ} \mathrm{C}$ for $1 \mathrm{~h}$ or OHTAM at $37^{\circ} \mathrm{C}$ for $1.5 \mathrm{~h}$, and the hemolysis was calculated from the absorbance of supernatants at $540 \mathrm{~nm}$ after centrifugation of erythrocyte suspension. The recordings obtained from the results expressed as percentage of total hemolysis are typical of three independent experiments.
1978), in agreement with the hydrophobicity, the high membrane partition and ordering effects of these compounds in lipid bilayer (Micol et al., 1990; Fukuzawa et al., 1992; Cruz-Silva et al., 2000), indicating that OHTAM-induced hemolysis is related with membrane interaction. Moreover, the fact that $\alpha$-TAc (with an inactivated functional hydroxyl and deprived of antioxidant activity) (Urano et al., 1992) inhibits also the hemolytic process suggests that the free hydroxyl group of $\alpha-T$ is not critical for protection against OHTAMinduced damage and that the hemolysis will be not caused by oxidative disruption of erythrocyte.

\subsection{Effect of OHTAM on oxidative stress of erythrocytes}

To exclude the putative involvement of peroxidative degradation mechanisms on the hemolytic effect of OHTAM, the oxygen consumption and hemoglobin oxidation were evaluated simultaneously in the same erythrocyte suspension during the time course of the drug-induced hemolysis. The hemolytic effect promoted by $35 \mu \mathrm{M}$ OHTAM, as followed by the increase in the hemoglobin released to the supernatant, is not accompanied by a significant decrease in $\mathrm{O}_{2}$ concentration in the medium (Fig. 4A) or changes in the spectra of the released oxyhemoglobin (Fig. 4B), in contrast to the abrupt $\mathrm{O}_{2}$ consumption (Fig. 4A) and conversion of the typical spectra of oxyhemoglobin into methemoglobin (Fig. 4C) induced by $20 \mathrm{~mm}$ AAPH, revealing an extensive peroxidation of lipids and proteins induced by the AAPH-derived radicals, as previously reported (Miki et al., 1987; Sato et al., 1995). Therefore, an oxidative
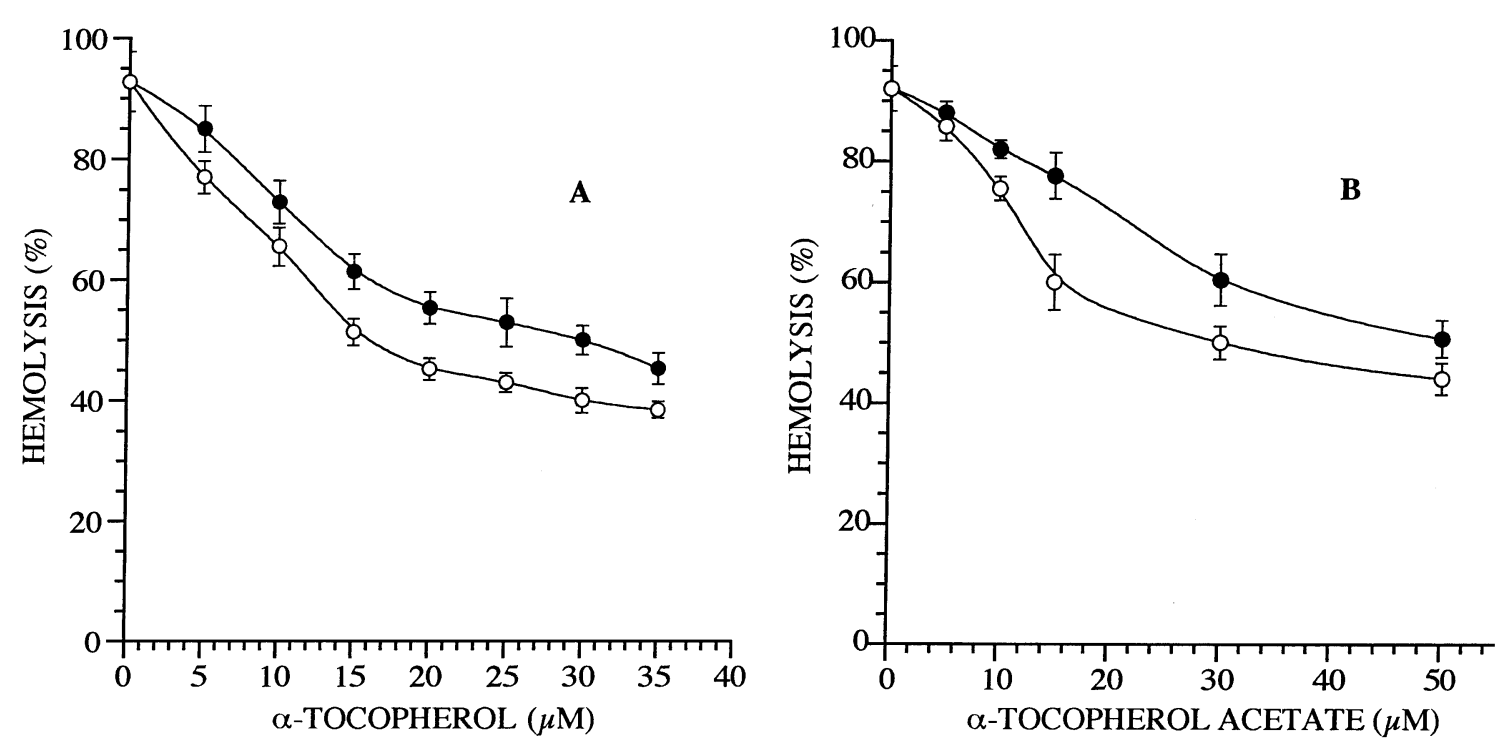

Fig. 3. Protection of $\alpha$-T (A) and $\alpha$-TAc (B) against OHTAM-induced hemolysis. The erythrocyte suspensions in PBS $(0.33 \%$ hematocrit) were preincubated with the tocopherols at $37^{\circ} \mathrm{C}$ for $2 \mathrm{~h}$ and afterwards the erythrocytes were washed $(\mathbf{O})$ or not $(\mathrm{O})$ prior to the addition of $35 \mu \mathrm{M}$ OHTAM, followed by incubation at $37^{\circ} \mathrm{C}$ for $90 \mathrm{~min}$. The hemolysis extension was estimated by the absorbance of supernatant at $540 \mathrm{~nm}$ as described in the legend to Fig. 1. Each point represents the mean \pm S.D. of three independent experiments. 
challenge of erythrocytes by the presence of OHTAM is not observed as compared with AAPH, a well-known reference oxidant (Miki et al., 1987; Niki, 1990; Sato et al., 1995). Moreover, the peroxidation process induced by AAPH-derived peroxyl radicals in erythrocyte suspensions, previously lysed with hypotonic PBS, is significantly inhibited by OHTAM, as assessed by $\mathrm{O}_{2}$ consumption (results not shown), pointing to the antioxidant activity of OHTAM in this biological system, as previously reported in other studies regarding the antioxidant mechanisms of OHTAM (Custódio et al., 1994; Wei et al., 1998).

\subsection{Osmotic behavior of erythrocytes in the presence of OHTAM}

The hemolytic action dependent on putative membrane perturbations was evaluated by the hemolytic fragility analysis as a function of $\mathrm{NaCl}$ concentration (Fig. 5). Incubation of the erythrocyte suspensions with $10 \mu \mathrm{M}$ OHTAM does not change the osmotic fragility profile, as compared to the control conditions. However, $20 \mu \mathrm{M}$ OHTAM, with no significant hemolytic effect in isotonic medium, considerably shifts the fragility profile. As already observed before, $35 \mu \mathrm{M}$ OHTAM induces total hemolysis independently of the osmolarity (data not shown). In contrast to TAM (Cruz-Silva et al., 2000), OHTAM increases the susceptibility of erythrocytes to hypotonic lysis, through changes in membrane permeability.

To further explore this permeabilizing effect, the efflux of intracellular $\mathrm{K}^{+}$in parallel with hemoglobin release was searched, since $\mathrm{K}^{+}$efflux has revealed a reliable methodology for the assay of permeability changes induced by several hemolytic compounds (Cybulska et al., 1995; Jackson et al., 1996).

Fig. 6 displays the $\mathrm{K}^{+}$and hemoglobin leakage curves resulting from the incubation of erythrocyte suspensions with $35 \mu \mathrm{M}$ OHTAM at $25^{\circ} \mathrm{C}$. Release of about $60 \%$ of intracellular $\mathrm{K}^{+}$occurs in a short period $(2 \mathrm{~min})$ and the remaining $\mathrm{K}^{+}$is gradually released after $20 \mathrm{~min}$ of incubation. In contrast, the release of hemoglobin to the
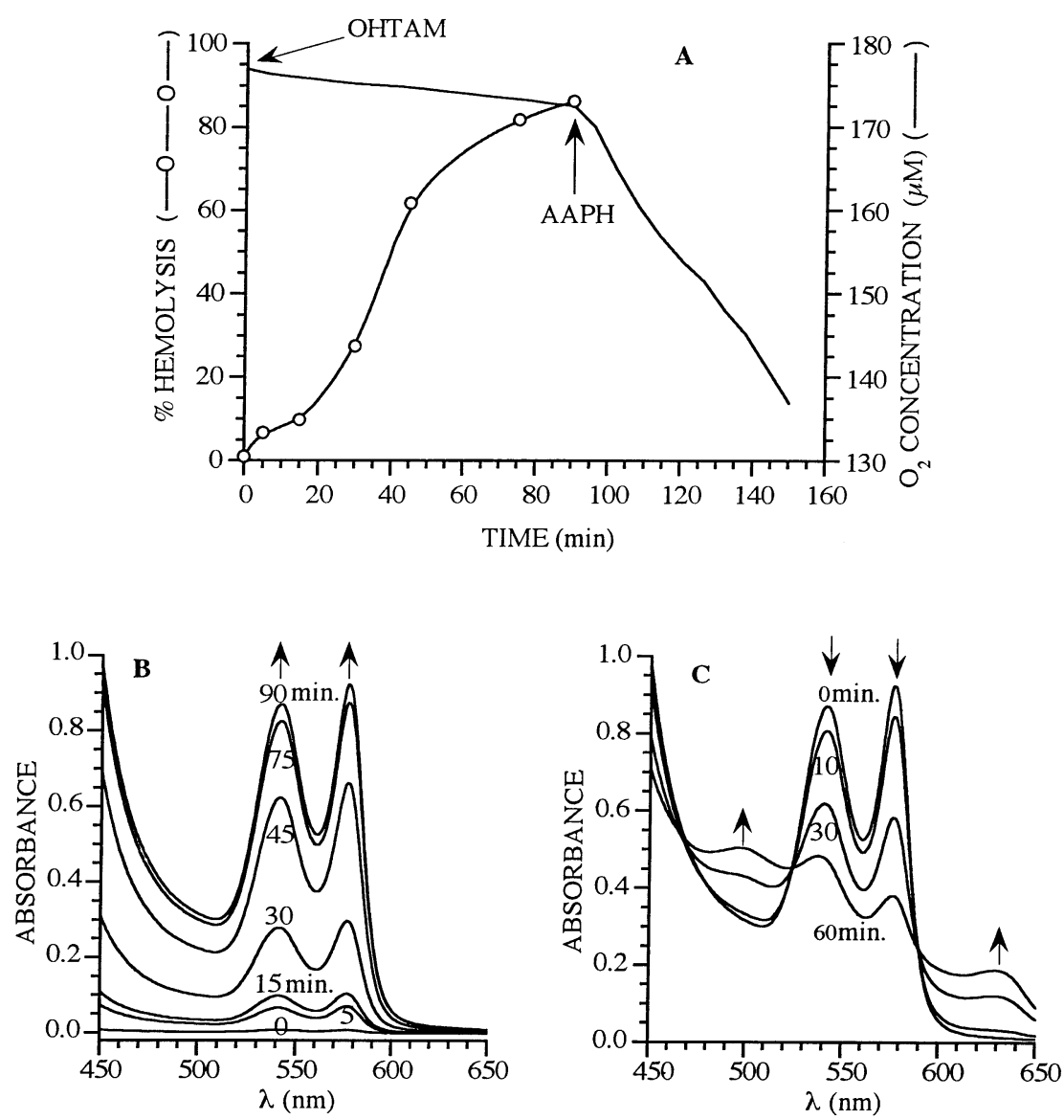

Fig. 4. Oxygen consumption rates $(-)$ associated with hemolysis of erythrocytes $(\bigcirc)$ incubated with $35 \mu$ M OHTAM and afterwards with 20 mM AAPH (A) and spectra of hemoglobin after the addition of OHTAM (B) and AAPH (C). $\mathrm{O}_{2}$ consumption (A) and hemoglobin spectra were monitored simultaneously during incubation of erythrocyte suspensions in PBS $\left(0.33 \%\right.$ hematocrit) with OHTAM at $37^{\circ} \mathrm{C}$ for $1.5 \mathrm{~h}(\mathrm{~B})$ and after AAPH addition (C), as described in Materials and Methods. Hemoglobin spectra were recorded at different reaction times indicated by the numbers adjacent to the traces. Upward and downward arrows indicate increase or decrease in absorbance due to hemoglobin release (B) and hemoglobin oxidation by AAPH added $1.5 \mathrm{~h}$ after OHTAM (C). The results are representative of three different assays. 
extracellular medium is a late phenomenon and total hemolysis is observed only after $90 \mathrm{~min}$ (Fig. 6). Therefore, OHTAM induces the early formation of permeability paths in the erythrocyte membrane, allowing the rapid efflux of $\mathrm{K}^{+}$prior to a large scale disruption of the membrane, which permits the release of hemoglobin.

\section{Discussion}

OHTAM interacts with human erythrocytes in vitro in a different way from that recently described for TAM (Cruz-Silva et al., 2000). The extension of OHTAM hemolytic effects depends on blood donor, being lower for older subjects (Fig. 1) as observed for TAM (CruzSilva et al., 2000). However, a complete hemolysis occurs only at $35 \mu \mathrm{M}$ OHTAM, incubated at $37{ }^{\circ} \mathrm{C}$ for $1.5 \mathrm{~h}$, independently of the erythrocyte donor, whereas $12.5 \mu \mathrm{M}$ TAM incubated at $37{ }^{\circ} \mathrm{C}$ for $1 \mathrm{~h}$ induces total hemolysis of erythrocytes (Fig. 2). Therefore, OHTAM requires a threefold higher concentration to induce the same hemolytic effect observed for TAM and the hemolysis takes longer, pointing out that OHTAM shows a much weaker hemolytic action than TAM. Considering that the tissue and serum concentrations of OHTAM are much lower as compared with TAM (Lien et al., 1987, 1991; MacCallum et al., 1996), OHTAM may not reach concentrations high enough to cause hemolysis, suggesting that this drug does not contribute to TAM-induced hemolytic anemia.

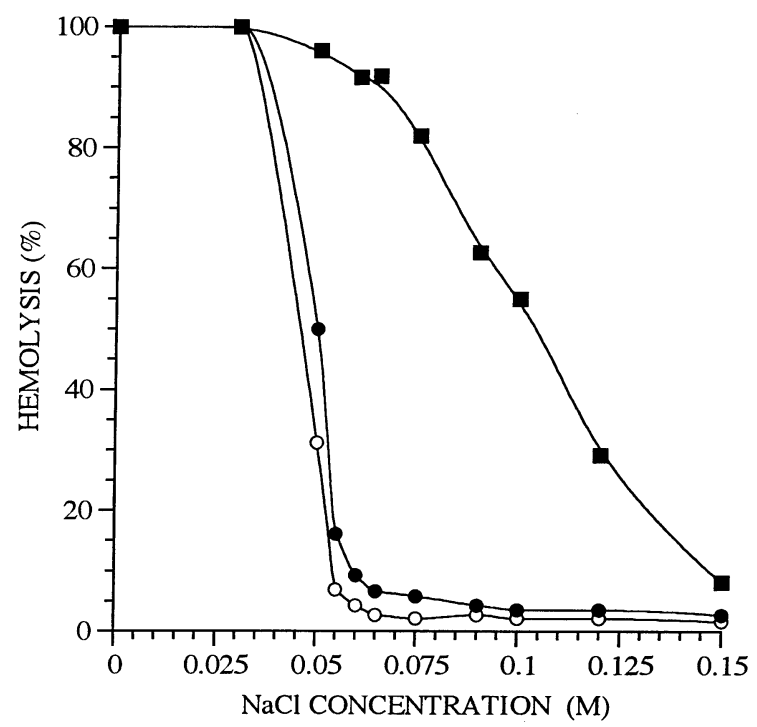

Fig. 5. Osmotic fragility curves of human erythrocytes as affected by the addition of OHTAM. The erythrocyte suspensions $(0.33 \%$ hema-

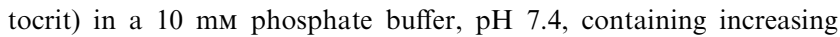
concentrations of $\mathrm{NaCl}$ were incubated at $37{ }^{\circ} \mathrm{C}$ for $1.5 \mathrm{~h}$ in the absence $(\bigcirc)$ and in the presence of $10(\bullet)$ and $20 \mu \mathrm{M}$ OHTAM $(\boldsymbol{\square})$. Hemolysis was estimated and expressed as described in the legend to the Fig. 1.
The inhibition of OHTAM-induced hemolysis by $\alpha$-T (Fig. 3A) could insinuate the oxidative damage of erythrocyte membrane as the mechanism involved in the hemolytic effect, since $\alpha$-T has a remarkable antioxidant capacity, interrupting free radical chain reactions in several membrane systems (Yamamoto and Niki, 1988; Ernster, 1993), including the human erythrocyte membrane (Yamamoto et al., 1985; Miki et al., 1987). However, $\alpha$-TAc, with its chromanol hydroxyl group acylated and thus deprived of antioxidant activity, also suppresses that hemolytic effect (Fig. 3B). Additionally, the hemolytic effect of OHTAM does not involve an increase in the membrane peroxidation or hemoglobin oxidative degradation (Fig. 4). On the other hand, OHTAM protects the peroxidative degradation of erythrocyte components induced by peroxyl radicals, according to its antioxidant properties in vitro (Custódio et al., 1994; Wei et al., 1998) and in vivo (Wei et al., 1998), acting as a potent intramembraneous scavenger of peroxyl radicals (Custódio et al., 1994). Therefore, OHTAM-induced hemolysis is not caused by the oxidative injury of the erythrocytes.

The effects of OHTAM on osmotic fragility and $\mathrm{K}^{+}$ release from erythrocytes are considerably different from those described for TAM (Cruz-Silva et al., 2000), and suggest different mechanisms of interaction with the erythrocyte membrane. TAM does not change the osmotic fragility curves of erythrocytes and shows only a weak prelytic release of $\mathrm{K}^{+}$from the cells (Cruz-Silva et al., 2000), whereas OHTAM strongly shifts the osmotic fragility profile and makes the red blood cells leaky to $\mathrm{K}^{+}$preceding the slower hemolysis process (Fig. 6), supporting the conclusion that OHTAM-

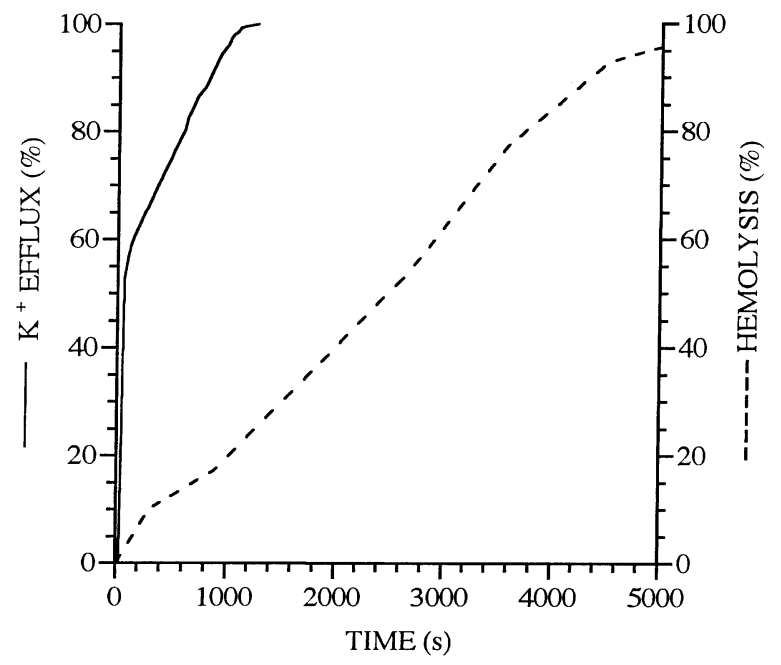

Fig. 6. Time courses of $\mathrm{K}^{+}(-)$and hemoglobin (---) release from human erythrocytes in $150 \mathrm{~mm} \mathrm{NaCl}, 10 \mathrm{~mm}$ sodium phosphate, $\mathrm{pH}=7.4(0.33 \%$ hematocrit $)$ incubated with $35 \mu \mathrm{M}$ OHTAM at $25^{\circ} \mathrm{C}$. The release of $\mathrm{K}^{+}$was estimated with a $\mathrm{K}^{+}$ion-sensitive electrode and hemolysis was evaluated by spectrophotometry of the supernatant at $540 \mathrm{~nm}$. The results are expressed as the percentage of total release in complete hemolysis and are typical of several independent assays. 
induced hemolysis occurs via an osmotic swelling. In fact, the osmotic hemolysis described for several compounds (Lieber et al., 1984; Deuticke et al., 1989; Jackson et al., 1996) involves the formation of permeability paths for small ions, which allow water osmosis as consequence of the increased osmotic pressure inside the erythrocyte. The osmotic imbalance causes cells to swell, inducing physical rupture of the membrane followed by hemoglobin leakage. OHTAM strongly partitions in biomembranes (Custódio et al., 1991) and distributes in the outer region of the lipid bilayer, mainly in the lipid-protein interfaces (Custódio et al., 1993b), which may account for membrane structural perturbations reflected in the permeability status (Figs. 5 and 6). The inhibitory effects of $\alpha$-T and $\alpha$-TAc towards OHTAM-induced hemolysis (Fig. 3) reflect a decrease of the permeability (Urano et al., 1992) or the exclusion of OHTAM from the membrane; that is, withdrawing the drug from the interaction with membrane components involved in the control of permeability due to the membrane stabilization induced by both tocopherols (Micol et al., 1990; Fukuzawa et al., 1992; Cruz-Silva et al., 2000).

In conclusion, OHTAM induces hemolysis, but to a much smaller extent as compared with TAM, ruling out this hemolytic effect in TAM-induced hemolytic anemia. Moreover, our findings suggest that, differently from those described for TAM (Cruz-Silva et al., 2000), OHTAM induces permeabilization of the erythrocyte membrane and a subsequent time-dependent hemolytic process, via an osmotic mechanism, occurring at relatively high concentrations. It is difficult to calculate precisely the concentrations of OHTAM resulting from pharmacological doses of TAM due to its high hydrophobic character, partitioning in biological membranes (Custódio et al., 1991) and high affinity to receptors in various target tissues (Borgna and Rochefort, 1980, 1981), which in humans is 10-60-fold higher in tissues than in serum (Lien et al., 1991). However, the concentrations of OHTAM that induce hemolysis $(>25 \mu \mathrm{M})$ are not easily reached in vivo in tissues and plasma, suggesting that little or no toxicity is expected with OHTAM doses as compared with TAM.

\section{Acknowledgements}

This work was supported by Sapiens99/36075/99 from Portuguese FCT. M. M. Cruz Silva was a recipient of a grant from Praxis XXI (Praxis XXI/BM/6569/95).

\section{References}

Araki, K., Rifkind, J.M., 1980. Erythrocyte membrane cholesterol: an explanation of the aging effect on the rate of hemolysis. Life Sciences 26, 2223-2230.
Borgna, J.L., Rochefort, H., 1980. High affinity binding to the estrogen receptor of $[3 \mathrm{H}] 4$-hydroxytamoxifen, an active antiestrogen metabolite. Molecular and Cellular Endocrinology 20, 71-85.

Borgna, J.L., Rochefort, H., 1981. Hydroxylated metabolites of tamoxifen are formed in vivo and bound to estrogen receptor in target tissues. Journal of Biological Chemistry 256, 859-868.

Ching, C.K., Smith, P.G., Long, R.G., 1992. Tamoxifen-associated hepatocellular damage and agranulocytosis. Lancet 339, 940.

Cruz-Silva, M.M., Madeira, V.M.C., Almeida, L.M., Custódio, J.B.A., 2000. Hemolysis of human erythrocytes induced by tamoxifen is related to disruption of membrane structure. Biochimica et Biophysica Acta 1464, 49-61.

Custódio, J.B.A., Almeida, L.M., Madeira, V.M.C., 1991. A reliable and rapid procedure to estimate drug partitioning in biomembranes. Biochemical and Biophysical Research Communications 176, 10791085.

Custódio, J.B.A., Almeida, L.M., Madeira, V.M.C., 1993a. The anticancer drug tamoxifen induces changes in the physical properties of model and native membranes. Biochimica et Biophysica Acta $1150,123-129$.

Custódio, J.B.A., Almeida, L.M., Madeira, V.M.C., 1993b. The active metabolite hydroxytamoxifen of the anticancer drug tamoxifen induces structural changes in membranes. Biochimica et Biophysica Acta $1153,308-314$.

Custódio, J.B.A., Almeida, L.M., Madeira, V.M.C., 1994. Tamoxifen and hydroxytamoxifen as intramembraneous inhibitors of lipid peroxidation. Evidence for peroxyl radical scavenging activity. Biochemical Pharmacology 47, 1989-1998.

Custódio, J.B.A., Almeida, L.M., Madeira, V.M.C., 1996. The effect of the anticancer drugs tamoxifen and hydroxytamoxifen on the calcium pump of isolated sarcoplasmic reticulum vesicles. Toxicology in Vitro 10, 523-531.

Custódio, J.B.A., Moreno, A.J.M., Wallace, K.B., 1998. Tamoxifen inhibits mitochondrial permeability transition pore opening induced by $\mathrm{Ca}^{2+}$ and inorganic phosphate. Toxicology and Applied Pharmacology 152, 10-17.

Cybulska, B., Bolard, J., Seksek, O., Czerwinski, A., Borowski, E., 1995. Identification of the structural elements of amphotericin B and other polyene macrolide antibiotics of the hepteane group influencing the ionic selectivity of the permeability pathways formed in the red cell membrane. Biochimica et Biophysica Acta 1240, 167-178.

Deuticke, B., Henseleit, U., Haest, C.W.N., Heller, K.B., Dubbelman, T.M.A.R., 1989. Enhancement of transbilayer mobility of a membrane lipid probe accompanies formation of membrane leaks during photodynamic treatment of erythrocytes. Biochimica et Biophysica Acta 982, 53-61.

Dwight, J.F., St, J., Hendry, B.M., 1996. The effects of tert-butyl hydroperoxide on human erythrocyte membrane ion transport and the protective actions of antioxidants. Clinica Chimica Acta 249, 167-181.

Ernster, L., 1993. Lipid peroxidation in biological membranes: mechanisms and implications. In: Yagi, K. (Ed.), Active Oxygen, Lipid Peroxides and Antioxidants. Japan Sci. Soc. Press, Tokyo, pp. 1-38.

Etienne, M.C., Milano, G., Fischel, J.L., Frenay, M., François, E., Formento, J.L., Gioanni, J., Namer, M., 1989. Tamoxifen metabolism: pharmacokinetics and in vitro study. British Journal of Cancer $60,30-35$.

Fukuzawa, K., Ikebata, W., Shibata, A., Kumadaki, I., Sakanaka, T., Urano, S., 1992. Location and dynamics of $\alpha$-tocopherol in model phospholipid membranes with different charges. Chemistry and Physics of Lipids 63, 69-75.

Jackson, J.K., Winternitz, C.I., Burt, H.M., 1996. Mechanism of hemolysis of human erythrocytes exposed to monosodium urate monohydrate crystals. Preliminary characterization of membrane pores. Biochimica et Biophysica Acta 128, 45-52.

Jordan, V.C., 1990. Long-term adjuvant tamoxifen therapy for breast cancer. Breast Cancer Research and Treatment 15, 125-136. 
Kemp, J.V., Adam, H.K., Wakeling, A.E., Slater, R., 1983. Identification and biological activity of tamoxifen metabolites in human serum. Biochemical Pharmacology 32, 2045-2052.

Koedijk, C.D.M.A., Blankenstein, M.A., Thijssen, J.H.H., 1994. Speculation on the mechanism of action of triphenylethylene antioestrogens. Biochemical Pharmacology 47, 1927-1937.

Koga, T., Moro, K., Terao, J., 1998. Protective effects of a vitamin E analog, phosphatidylchromanol, against oxidative hemolysis of human erythrocytes. Lipids 33, 589-595.

Lieber, M.R., Lange, Y., Weinstein, R.S., Steck, T.L., 1984. Interaction of chlorpromazine with the human erythrocyte membrane. Journal of Biological Chemistry 259, 9225-9234.

Lien, E.A., Ueland, P., Solheim, E., Kvinnsland, S., 1987. Determination of tamoxifen and four metabolites in serum by lowdispersion liquid chromatography. Clinical Chemistry 33, 16081614.

Lien, E.A., Solheim, E., Ueland, P.M., 1991. Distribution of tamoxifen and its metabolites in rat and human tissues during steady-state treatment. Cancer Research 51, 4837-4844.

Lopes, M.C.F., Vale, M.G.P., Pato, A.P., 1990. $\mathrm{Ca}^{2+}$-dependent binding of tamoxifen to calmodulin isolated from bovine brain. Cancer Research 50, 2753-2758.

MacCallum, J., Cummings, J., Dixon, J.M., Miller, W.R., 1996. Solidphase extraction and high-performance liquid chromatographic determination of tamoxifen and its major metabolites in plasma. Journal of Chromatography B 678, 317-323.

McCague, R., Parr, I.B., Leclerq, G., Leung, O.T., Jarman, M., 1990. Metabolism of tamoxifen by isolated rat hepatocytes. Biochemical Pharmacology 39, 1459-1465.

Micol, V., Aranda, F.J., Villalain, J., Gómez-Férnandez, J.C., 1990. Influence of vitamin $\mathrm{E}$ on phosphatidylethanolamine lipid polymorphism. Biochimica et Biophysica Acta 1022, 194-202.

Miki, M., Tamai, H., Mino, M., Yamamoto, Y., Niki, E., 1987. Freeradical chain oxidation of rat red blood cells by molecular oxygen and its inhibition by $\alpha$-tocopherol. Archives of Biochemistry and Biophysics 258, 373-380.

Nayfield, S.G., Karp, J.E., Ford, L.G., Dorr, F.A., Kramer, B.S., 1991. Potential role of tamoxifen in prevention of breast cancer. Journal of the National Cancer Institute 83, 1450-1459.

Niki, E., 1990. Free radicals as source of water- or lipid-soluble peroxyl radicals. Methods in Enzymology 186, 100-109.

Oosbree, T.R.V., Olsen, M.R., Tate, A.C., Jordan, V.C., Mueller,
G.C., 1984. An immobilized antiestrogen binds a specific uterine protein in addition to estrogen receptor proteins. Molecular and Cellular Endocrinology 35, 143-149.

Sato, Y., Kamo, S., Takahashi, T., Suzuki, Y., 1995. Mechanism of free radical-induced hemolysis of human erythrocytes: hemolysis by water-soluble radical initiator. Biochemistry 34, 8940-8949.

Schuck, P., Schubert, D., 1991. Band 3-hemoglobin associations-the band 3 tetramer is the oxyhemoglobin binding site. FEBS Letters 293, 81-84

Shinitzky, M., Barenholz, Y., 1978. Fluidity parameters of lipid regions determined by fluorescence polarization. Biochimica et Biophysica Acta 515, 367-394.

Sica, G., Natoli, C., Marchetti, P., Piperno, S., Iacobelli, S., 1984. Tamoxifen induced membrane alterations in human breast cancer cells. Journal of Steroid Biochemistry and Molecular Biology 20, 425-428.

Takeuchi, Y., Yamaoka, Y., Morimoto, Y., Kaneko, I., Fukumori, Y., Fakuda, T., 1989. Stabilizing effects of some amino acids on membranes of rabbit erythrocytes perturbed by chlorpromazine. Journal of Pharmaceutical Sciences 78, 3-7.

Thangaraju, M., Ezhilarasi, R., Sachdanandam, P., 1995. Effect of tamoxifen on erythrocyte membrane lipids, lipid peroxides, and antioxidant enzymes in breast cancer women. Cancer Biochemistry and Biophysics 14, 297-302.

Urano, S., Inomoyi, Y., Sugawara, T., Kato, Y., Kitahara, M., Hasegawa, Y., Matsuo, M., Mukai, K., 1992. Vitamin E: Inhibition of retinol-induced hemolysis and membrane-stabilizing behavior. Journal of Biological Chemistry 267, 18365-18370.

Vogel, S.S., Beushausen, S., Lester, D.S., 1995. Application of a membrane fusion assay for rapid drug screening. Pharmaceutical Research 12, 1417-1422.

Wei, H., Cai, Q., Tian, L., Lebwohl, M., 1998. Tamoxifen reduces endogenous and UV light-induced oxidative damage to DNA, lipid and protein in vitro and in vivo. Carcinogenesis 19, 1013-1018.

Yamamoto, K., Niki, E., Eguchi, J., Kamiya, Y., Shimasaki, H., 1985. Oxidation of biological membranes and its inhibition. Free radical chain oxidation of erythrocyte ghost membranes by oxygen. Biochimica et Biophysica Acta 819, 29-36.

Yamamoto, K., Niki, E., 1988. Interaction of $\alpha$-tocopherol with iron: antioxidant and prooxidant effects of $\alpha$-tocopherol in the oxidation of lipids in aqueous dispersions in the presence of iron. Biochimica et Biophysica Acta 958, 19-23. 